\section{Neoplasias de glândulas salivares: estudo de 119 casos}

\author{
Salivary gland neoplasms: a study of 119 cases
}

Gilda da Cunha Santos ${ }^{1}$

Marcos Roberto Martins ${ }^{2}$

Lucila Böheme Pellacani ${ }^{3}$

Ana Cristina Trench Vieira ${ }^{3}$

Luiz Augusto Nascimento ${ }^{4}$

Márcio Abrahão 5

\section{unitermos resumo}

Glândulas salivares

Objetivo: Revisão dos achados clinicopatológicos dos tumores de glândulas salivares diagnosticados

Tumores
Universidade Federal de São Paulo (EPM/Unifesp). Método: Foi realizado estudo retrospectivo de

205.967 diagnósticos anatomopatológicos e encontrados 119 casos de neoplasias de glândulas salivares, sendo 93 (78\%) neoplasias benignas e 26 (22\%) malignas. Resultados: A idade variou de 6 a 74 anos (média de 40 anos), sendo 71 (60\%) pacientes do sexo feminino e 48 (40\%) do sexo masculino. Com relação à topografia, $84(70,6 \%)$ casos foram de parótida, 23 (19,3\%) de glândula submandibular, $11(9,24 \%)$ de glândulas salivares menores e um $(0,86 \%)$ de glândula sublingual. Quanto ao tipo histológico, os tumores corresponderam a 59,7\% de adenomas pleomórficos, 10\% de cistoadenomas papilíferos linfomatosos (tumor de Warthin), 9,3\% de carcinomas adenóides císticos e 8,4 \% de carcinomas mucoepidermóides. Conclusão: O padrão de distribuição dos tumores de glândulas salivares foi semelhante ao encontrado em outras séries. A análise desta distribuição permite identificar variáveis como localização e padrão histológico, subsídios importantes que devem ser considerados no tratamento e em estudos de marcadores prognósticos.

\section{abstract}

Background: The purpose of this study was to review clinicopathological aspects of salivary glands tumours from 1980 through 1997 at Departament of Pathology of Paulista School of Medicine - Federal University of São Paulo - Unifesp. Methods: We retrospectively evaluated 205.967 pathological reports and we found 119 salivary gland tumours, 93 benign neoplasms (78\%) and 26 malignant tumours (22\%). Results: The age range was 6-74 years (mean age 40 years), 71 cases were in female (60\%) and 48 cases were in male (40\%). The distribution of tumours in various sites were 84 cases of parotid tumours (70.6\%), 23 cases of submandibular (19.3\%), 11 cases of minor (oropharyngeal) glands (9.24\%) and just one case of sublingual gland tumour (0.86\%). Pleomorphic adenoma forming $59.7 \%$ of the total salivary gland tumours, Warthin's tumour forming $10 \%$ of the series, adenoid cystic carcinoma forming 9.3\% and mucoepidermoid carcinoma forming 8.4\%. Conclusions: Some aspects like distribution of tumour in relation to the site and histopathological pattern are very important to considered in the treatment of the disease and prognosis.
1. Professora-adiunta do Departamento de Patologia da Escola

Paulista de Medicina da Universidade Federal de São Paulo (EPM/Unifesp). 2. Pós-graduando (mestrado), Departamento de Patologia da EPM/Unifesp.

3. Aluna do curso de

Medicina; bolsista de Iniciação

Cientifica do CNPq -

Departamento de Patologia da EPM/Unifesp.

4. Pós-graduando do

Departamento de Otorrinolaringologia e Cirurgia de Cabeça e Pescoço da EPM/Unifesp. 5. Professor adiunto do Departamento de Otorrinolaringologia e Cirurgia de Cabeça e Pescoço da EPM/Unifesp. Trabalho realizado no Departamento de Patologia da EPM/Unifesp.

Trabalho apresentado no VII Congresso de Iniciação Científica Unifesp e no XXII Congresso Brasileiro de Patologia. 


\section{Introdução}

As neoplasias de glândulas salivares são incomuns e perfazem cerca de $2 \%$ a $6,5 \%$ dos tumores da região da cabeça e pescoço. Considerando a ampla variedade de comportamentos biológicos e tipos histológicos que estes tumores apresentam, a abordagem deste tema torna-se um desafio.

A baixa incidência das neoplasias de glândulas salivares é um dos principais fatores determinantes para que vários trabalhos enfatizem a análise retrospectiva, considerando o local de incidência, o tipo histológico e a forma de tratamento.

Vários trabalhos mostram o padrão de comportamento das neoplasias de glândulas salivares. Alguns autores avaliaram os aspectos biológicos envolvidos em tumores de glândulas salivares menores $(8,9,17,19)$. Outros estudos levantaram fatores coadjuvantes no comportamento dos carcinomas de glândulas salivares, apontando as glândulas salivares maiores, principalmente a parótida, como principal sítio da lesão $(4,18,23)$. Foi relatada influência substancial do tipo histológico em relação à localização do tumor (3). Portanto, o padrão de distribuição dos tumores de glândulas salivares é fator importante que deve ser considerado na avaliação do comportamento biológico destas neoplasias.

Este trabalho teve por objetivo a revisão de 119 casos de tumores de glândulas salivares, considerando os achados histopatológicos e os dados clínicos, confrontando estes resultados com os encontrados na literatura.

\section{Material e método}

Foi realizado estudo retrospectivo das neoplasias de glândulas salivares diagnosticadas no período de 1980 a 1997 no Departamento de Patologia da Escola Paulista de Medicina da Universidade Federal de São Paulo (EPM/ Unifesp). Os diagnósticos foram revistos segundo a classificação dos tumores de glândulas salivares da OMS (1992). Os dados clínicos de idade, sexo e topografia foram obtidos a partir dos laudos anatomopatológicos.

\section{Resultados}

Do total de 205.967 diagnósticos anatomopatológicos realizados nesse período foram encontrados 119 casos de neoplasias de glândulas salivares. A idade dos pacientes variou de 6 a 74 anos. A média de idade foi de 40 anos.
De acordo com a Tabela 1, dos 119 espécimes cirúrgicos foram observados 93 casos benignos (78\%) e 26 malignos (22\%). A média de idade dos pacientes com tumores benignos foi de 47 anos e de 45 anos para aqueles com tumores malignos. Entre os benignos, conforme a Tabela 2, a distribuição foi a seguinte: adenoma pleomórfico $(76,3 \%)$, cistoadenoma papilífero linfomatoso (13\%), adenoma canalicular (2\%), adenoma de células basais variante trabecular (2\%), outros (oncocitoma e mioepitelioma) (6,7\%). Para os malignos (Tabela 3) observaram-se: carcinoma adenóide cístico $(42,3 \%)$, carcinoma mucoepidermóide (38,4\%), carcinoma de células acinares $(7,7 \%)$, outros (carcinoma de ductos, adenocarcinoma polimorfo de baixo grau e carcinoma de células basais) (11,6\%). Dos 119 casos, 93 (60\%) eram pacientes do sexo feminino e 48 (40\%) do sexo masculino, conforme a Tabela 4. Na Figura, as faixas etárias que apresentaram o maior número de casos foram as compreendidas entre 31-40 anos, 21-30 e 51-60. A Tabela 5 apresenta a distribuição topográfica, sendo a parótida o sítio mais freqüente, com 84 casos $(70,6 \%)$, seguida por 23 casos da glândula submandibular (19,3\%), 11 de glândulas salivares menores $(9,24 \%)$ e um caso de glândula sublingual $(0,86 \%)$. Distribuição semelhante foi observada para ambos os sexos.

Tabela 1 e malignos

Distribuição do total de tumores de glândulas salivares benignos

\begin{tabular}{lcc} 
& № de casos & $(\%)$ \\
Malignos & 26 & 22 \\
Benignos & 93 & 78 \\
Total & 119 & 100 \\
\hline
\end{tabular}

Distribuição da porcentagem de tumores benignos de glândulas

Tabela 2 salivares segundo o tipo histológico

Classificação histológica

$(\%)$

Adenoma pleomórfico

Cistoadenoma papilífero linfomatoso

Adenoma canalicular

Adenoma de células basais variante trabecular 2

Outros

Total 
Distribuição da porcentagem de tumores malignos de glândulas

Tabela 3 salivares segundo o tipo histológico

\section{Classificação histológica}

Carcinoma adenóide cístico 42,3

Carcinoma mucoepidermóide

38,4

Carcinoma de células acinares

7,7

Outros

11,6

Total

100

\section{Distribuição do total de casos}

\section{de tumores de glândulas salivares}

Tabela 4 por sexo

\begin{tabular}{lcc}
\hline Sexo & Número & $(\%)$ \\
Masculino & 48 & 40 \\
Feminino & 71 & 60 \\
Total & 119 & 100 \\
\hline
\end{tabular}

\section{Tabela 5 por topografia}

\section{Distribuição do total de casos de tumores de glândulas salivares}

\begin{tabular}{lcc}
\hline Sítio de origem & Número de casos & $(\%)$ \\
Parótida & 84 & 70,6 \\
Glândula submandibular & 23 & 19,3 \\
Glândulas salivares menores & 11 & 9,24 \\
Glândula sublingual & 1 & 0,86 \\
Total & 119 & 100 \\
\hline
\end{tabular}

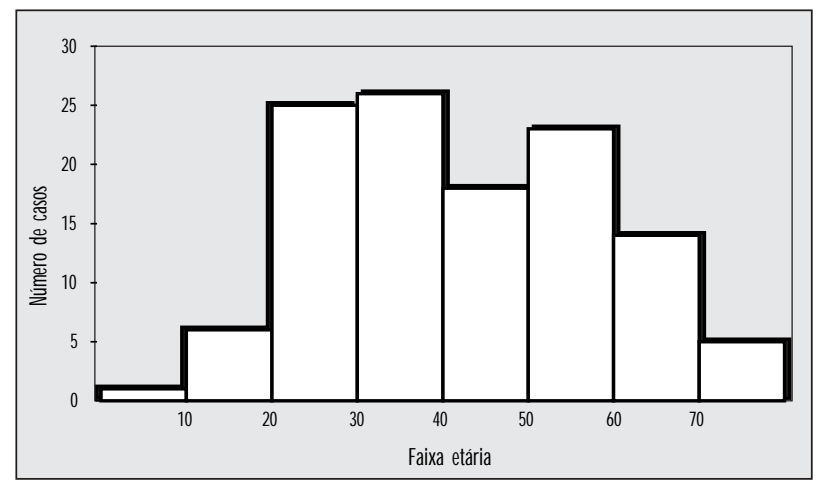

Figura - Distribuição por faixa etária e idade média dos casos encontrados

\section{Discussão}

Este trabalho baseou-se na distribuição amostral de tumores benignos e malignos de glândulas salivares. Ao se considerar estudos sobre neoplasias de glândulas salivares, poucos são os trabalhos que abordam tanto as glândulas salivares maiores quanto as menores da cavidade bucal. Muitos têm como referência a incidência em um dos dois tipos de glândula. Analisando tumores em glândulas salivares menores, foi relatado que dos 106 casos diagnosticados, $55 \%$ eram tumores benignos, enquanto $45 \%$, malignos (9). Semelhante distribuição foi encontrada por outros autores (19) (57,5\% benignos e 42,5\% malignos). Outros trabalhos demonstraram uma diferença na distribuição entre tumores de glândulas salivares menores, sendo aproximadamente $65 \%$ tumores benignos e $35 \%$ malignos $(5,17)$. Esta diferença mostra-se ainda maior quando se consideram as glândulas salivares maiores e menores em conjunto $(13,20)$, como comprovam os resultados obtidos neste estudo ( $78 \%$ benignos e $22 \%$ malignos).

Ao considerar o tipo de tumor benigno, o diagnóstico de adenoma pleomórfico foi observado em $76,3 \%$ dos casos. A maior distribuição deste tipo de neoplasia benigna está em concordância com o encontrado em outras séries $(7-9,17,19)$. Em relação aos tumores malignos, os dados obtidos revelaram que o carcinoma adenóide cístico e o carcinoma mucoepidermóide foram os mais encontrados, havendo uma maior freqüência do primeiro tipo (42,3\% e $38,4 \%$, respectivamente), sendo a distribuição semelhante à encontrada por outros autores $(8,11,17$, $19,23)$. Contudo outros estudos evidenciaram maior incidência de carcinoma mucoepidermóide em relação ao adenóide cístico $(9,16,18,22)$. A avaliação da proliferação celular de carcinoma mucoepidermóide por meio de estudo imunoistoquímico tem sido utilizada como auxiliar na classificação dos tumores e na determinação do prognóstico (1). Ao se considerar recorrência da lesão versus tumor maligno primário, observou-se que na glândula parótida o carcinoma adenóide cístico prevaleceu sobre o carcinoma mucoepidermóide (21). Análise multivariada de sobrevida de 128 casos de carcinoma de glândulas salivares menores mostrou maior porcentagem de carcinoma mucoepidermóide $(59,4 \%)$, sendo o palato duro a localização mais afetada (48,4\%) (6).

Notou-se uma preponderância do sexo feminino, concordando com outros estudos $(4,17,19)$ que também mostram distribuição de 3:2. Quando se compararam a relação entre sexo (masculino e feminino) e o tipo de 
tumor (maligno e benigno), não foi encontrada diferença no padrão de distribuição, semelhante a outros resultados (9).

Considerando a relação entre localização do tumor e sexo, a distribuição foi semelhante para todos os sítios. Os resultados demonstraram também que a faixa etária de maior incidência foi entre 31 e 40 anos.

Em relação à distribuição da lesão, os dados obtidos evidenciaram que a parótida foi o sítio mais freqüentemente afetado (70,6\%), enquanto as glândulas salivares menores corresponderam a $9,24 \%$ dos casos. Semelhante distribuição também foi encontrada por outros autores (13). Mesmo nos casos de carcinomas incomuns que acometem os ductos, o sítio mais afetado é a parótida (10). Numa análise de apenas carcinomas de glândulas salivares verificou-se distribuição semelhante entre glândula parótida e glândula salivar menor $(44,4 \%$ e $38,3 \%$, respectivamente) (22).

O exame por congelação para os tumores da glândula salivar empregado isoladamente não é suficiente para decidir a melhor conduta, e sua interpretação deve ser feita correlacionando-se os achados clínicos e intra-operatórios (2). A variabilidade morfológica, a complexidade e a superposição de padrões histológicos dos tumores de glândulas salivares refletem-se no material citológico obtido por punção aspirativa, tornando-se difícil adquirir expertise nessa área (12).

A proposta de um sistema de estadiamento clínico baseado no tamanho da lesão primária, a presença de fixação ou ulceração, o envolvimento do nervo facial e do nódulo linfático cervical parecem auxiliar no tratamento da lesão (15). Este sistema de estadiamento clínico proposto também pode ser utilizado para glândulas submandibulares (14).

O padrão de distribuição de tumores de glândulas salivares é um subsídio importante que deve ser considerado no tratamento, na avaliação do prognóstico e em estudos de marcadores biológicos destas neoplasias.

\section{Conclusões}

Considerando a metodologia empregada e os resultados obtidos na revisão dos casos, pode-se concluir que houve uma maior incidência de tumores benignos em relação aos malignos. Entre os tumores benignos, o adenoma pleomórfico foi o tipo mais encontrado, e o sítio mais afetado foi a glândula parótida. A inter-relação de análise histopatológica e dados clínicos pode ser considerada fator preponderante no tratamento e na avaliação do prognóstico destas neoplasias.

\section{Referências}

1. Cardoso,W .P. et al. Proliferating cell nuclear antigen expression in mucoepidermoid carcino ma of salivary glands. São Paulo Med. J., 118: 69-74, 2000.

2. Carvalho, M.B. et al. Perioperative frozen section examination in parotid gland tumors. São Paulo Med. J., 117: 233-7, 1999.

3. C hilla, R. et al. M alignant salivary glands tumors. Effect of histolo gy and site on prognosis. HN 0, 31:286-90, 1983.

4. Kalmovich, B.; Kuten, A \& Robinson E. Malignant salivary gland tumors. Harefuah, 129: 308-12, 1995.

5. Lopes, M.A. et al. A clinicopathologic study of 196 intraoral minor salivary gland tumours. J. Oral Pathol. M ed., 28: 2647, 1999.

6. Lopes, M.A.; Santos, G.C . \& Kowalski, L.P. Multivariate survival analysis of 128 cases of oral cavity minor salivary gland carcinomas. Head \& N eck, 20:699-706, 1998.

7. Loyola,A.M . et al. Minor salivary gland tumours. A retrospective study of 164 cases in a Brazilian po pulation. Eur.J. Cancer B. Oral Oncol., 31B: 197-201, 1995.

8. Ma, D.Q. \& Yu Gy. Tumours of the minor salivary glands. A clinicopathologic study of 243 cases. Acta Otolaryngol.,103: 325-31, 1987
9. N eely, M.M.; Rohrer, M.D. \& Yong SK.Tumors of minor salivary glands and the analysis of 106 cases.J. 0 kla Dent.Assoc., 86 : 50-2,1996.

10.Ruíz-G odoy,R.L. et al. U nusual salivary glands adenocarcino mas: a retrospective study. J. Exp. Clin. Res., 20: 189-94, 2001.

11. Sadeghi, A. et al. Minor gland tumours of head and neck: treatment strategies and prognosis. Am.J. Clin. Oncol., 16:38, 1993.

12. Schindler, S. et al. D iagnostic challenges in aspiration cytolo gy of the salivary glands. Semin. Diagn. Pathol.,18:124-46,2001.

13. Spiro, R. Salivary neo plasms: overview of 35 years experience with 2,807 patients. Head N eck Surg., 8: 177-84,1986.

14. Spiro, R.; Hajdu, S. \& Strong W. Tumors of the submaxillary gland. AJS, 132:463-8, 1976

15. Spiro, R.; H uvos, A.G . \& Strong, E.W . Cancer of the parotid: a clinical pathologic study of 288 primary cases. AJS, 130: 452-9, 1975.

16. Spitz, R.M . \& Batsakis, G .J. Major salivary gland carcino ma. Arch Otolaryngol., 110:45-9, 1984.

17. Takahashi, H. et al. Intraoral minor salivary gland tumors: a demographic and histologic study of 200 cases. Tohoku J. Exp. Med., 161:111-28, 1990. 
18. Tran, L. et al. Major salivary gland tumors: treatment results and prognosis factors. Laryngoscope, 96: 1139-44,1986.

19. W aldron, C .A.; Mofty, S.K. \& Gnepp, D.R. Tumors of the intrao ral minor salivary glands:a demografic and histologic study of 426 cases. Oral Surg. Med. Oral Pathol., 66: 32333, 1988.

20. W illiams, N .P. et al.Salivary gland lesions: a Jamaican perspective. West Indian Med. J., 50:62-5, 2001.
21. W oods,J.E.; C hong, G.C .\& Beahrs, O .H .Experience with 1,360 primary parotid tumors. AJS, 130:460-2, 1975.

22. Yu, G .Y. \& Ma, D.Q . Carcinoma of the salivary glands: a clinical analysis of 342 cases. Chung Hua Chung Liu Tsa Chih, 8: 395-8, 1986.

23. Yu, G.Y. \& Ma, D.Q. Carcinoma of the salivary glands: a clinico pathologic study of 405 cases. Semin. Surg. Oncol., 3: 240-4, 1987. 